EPJ Web of Conferences 49, 14006 (2013)

DOI: 10.1051/epjconf/20134914006

(C) Owned by the authors, published by EDP Sciences, 2013

\title{
Diboson productions and aTGCs search at LHC
}

\author{
Yusheng $\mathrm{Wu}^{1,2, \mathrm{a}}$, on behalf of ATLAS and CMS collaborations \\ ${ }^{1}$ University of Michigan \\ ${ }^{2}$ University of Science and Technology of China
}

\begin{abstract}
The ATLAS and CMS collaborations have measured the production cross-sections of dibosons ( $W W$, $W Z, Z Z, W \gamma, Z \gamma)$ in leptonic decay final states with collision data produced at the LHC with both $\sqrt{s}=7 \mathrm{TeV}$ and $\sqrt{s}=8 \mathrm{TeV}$. Based on these data, limits on anomalous triple-gauge-couplings are also derived.
\end{abstract}

\section{Introduction}

The Standard Model (SM) electroweak theory is based on the $S U(2)_{L} \otimes U(1)_{Y}$ gauge groups, in which the gauge symmetry is spontaneously broken by the Higgs mechanism. The studies of diboson productions at LHC help to test the theory at $\mathrm{TeV}$ scale, improve the understanding of background processes in Higgs searches and also offer great opportunities to search for new physics at the high energy frontier.

At leading order, dibosons are produced mainly through $q \bar{q}$ annihilation, while $g g$ fusion contributes typically less than $10 \%$ [3]. The diboson productions of $W W$, $W Z, Z Z$ and $W / Z+\gamma$ are studied in leptonic decay final states. Diboson production cross-sections are known to next-to-leading order (NLO) in QCD, and the electroweak radiation corrections to the cross-sections are important at high $\sqrt{s}$ [4]; besides, the leptonic decay final states offer clean signature and enable the possibility of precision measurements. These cross-section measurements therefore provide crucial and sensitive test of both QCD and electroweak theory at the $\mathrm{TeV}$ scale.

Many new physics models predict resonance particles that can decay to boson pairs. Some of these models are based on the non-SM implementation of electroweak symmetry breaking; for instance, there are little Higgs [5], technicolor [6], extended gauge models [7] and RandallSundrum graviton [8] models. These new particles can be searched in either fully leptonic or semileptonic decay final states of diboson events, and the high energy of the LHC offers the greatest potential. A direct search for new resonances is not covered in this paper, instead an indirect approach is carried out by searching for anomalous triple-gauge-couplings (aTGCs). In the SM, triple-gaugecouplings (TGCs) are completely determined by gauge theory; and any deviation from the SM couplings indicates existence of new physics beyond the SM. aTGCs depend strongly on the center of mass energy of the colliding

\footnotetext{
a e-mail: Yusheng.Wu@cern.ch
}

bosons; therefore, the LHC offers a great opportunity for measuring stringent limits. Besides, aTGCs largely manifest themselves in high momentum or high mass regions, the sensitivity can be further enlarged by proper binning of kinematics distributions.

The reported analysis results are based on the $p p$ collision data collected by the ATLAS [1] and CMS detectors [2] during the years of 2011 and 2012. The integrated luminosities of analyzed data are slightly different among various diboson channels; but the maximum amount of data in use corresponds to about $5 \mathrm{fb}^{-1}$ at both $\sqrt{s}=7 \mathrm{TeV}$ and $\sqrt{s}=8 \mathrm{TeV}$. The detectors were operating amazingly well during the data taking periods; the detectors operated at a fraction of above $95 \%$ operational channels and data taking efficiencies were higher than $93 \%$. The following sections will describe both the results of cross-section measurements and aTGCs search.

\section{Cross-section measurement}

\subsection{Overview}

As an overview, Figure 1 shows the measured production cross-sections of various diboson processes and their comparisons with SM NLO predictions from the ATLAS and CMS experiments $[9,10]$. The measurements are consistent with theoretical predictions.

\section{$2.2 W / Z+\gamma$}

In this channel, events with a leptonic decay boson ( $W$ or $Z$ ) and a photon are selected. Major background processes include $W / Z+$ jets and $\gamma+$ jets, where jet-induced photons or jet-faking leptons are selected. In order to suppress these backgrounds, events with low boson mass or low $E_{\mathrm{T}}^{\text {miss }}$ are rejected, and in addition photons are required to be separated from leptons. The total systematic uncertainty for the measured cross-section is about $7-9 \%$; the major ones come from photon reconstruction and the background estimation. The cross-sections are measured 
in bins of the transverse photon energy $\left(E_{\mathrm{T}}^{\gamma}\right)$, and presented in Figure $2[11,12]$.

\section{$2.3 W W$}

$W W$ events are selected to have two high $p_{\mathrm{T}}$ isolated leptons and large $E_{\mathrm{T}}^{\mathrm{miss}}$. Backgrounds consist of $t \bar{t}, Z+$ jets, $W+$ jets and other diboson processes. The signal is purified by removing events within $\mathrm{Z}$ mass windows, rejecting events with one or more jets and discarding low $p_{\mathrm{T}}^{\ell \ell}$ (transverse momentum of two lepton system) region. The dominant source of uncertainty is the jet-veto uncertainty, and the total systematic uncertainty is around $8 \%$. Measured cross-sections are in agreement with theory, which are presented in Table $1[13,14]$.

\section{$2.4 W Z$}

Three leptons plus large $E_{\mathrm{T}}^{\text {miss }}$ make this signature very clean, but there are still background events contributing from $Z Z, Z+$ jets and $t \bar{t}$. An on-shell $\mathrm{Z}$ and a $\mathrm{W}$ boson with large transverse mass are required in these events in order to further suppress the contamination. The systematic uncertainties mainly come from lepton and $E_{\mathrm{T}}^{\text {miss }}$ reconstructions, which turns out to be around $5 \%$. The measured cross-sections are shown in Table $2[15,16]$.

\section{$2.5 W W / W Z \rightarrow \ell v j j$}

In this analysis, the semi-leptonic decays of diboson are studied. The signal events have one lepton, large $E_{\mathrm{T}}^{\text {miss }}$ and two separated jets, where the jets are from hadronic $\mathrm{W}$ or $\mathrm{Z}$ boson decay. The irreducible $W+$ jets background is subtract using template fit method, and the combined cross-section of $W W+W Z$ is derived as shown in Table $3[17,18]$, where the large systematic uncertainty is driven by uncertainties in the background estimation.

\section{$2.6 \mathrm{ZZ}$}

The $Z Z$ production cross-section is measured in two decay modes: $Z Z \rightarrow 4 \ell$ and $Z Z \rightarrow \ell \ell v v$. In the four lepton case, the signature is very clean with background contribution of less than $2 \%$ after requiring two on-shell $Z$ bosons for the signal selection. The measured cross-sections in both ATLAS and CMS are shown in Table $4[19,20]$. In the two lepton plus $E_{\mathrm{T}}^{\mathrm{miss}}$ channel, there is large background contamination from $Z+j e t s, t \bar{t}$ and other diboson processes. Apart from selecting an on-shell Z, events are further required to have zero jets, large $E_{\mathrm{T}}^{\text {miss }}$ in the $\mathrm{Z}$ transverse momentum direction. The measured cross-section in ATLAS at $\sqrt{s}=7 \mathrm{TeV}$ is $5.4_{-1.2}^{+1.3}$ (stats. $)_{-1.0}^{+1.4}$ (syst.) \pm 0.2 (lumi.) $\mathrm{pb}$ [21], which is consistent with SM NLO prediction.

\section{3 aTGCs search}

The search is generally based on kinematics distribution of the boson $p_{\mathrm{T}}$ or the mass of the diboson system. Then likelihood functions are constructed and systematic uncertainties are incorporated as nuisance parameters. The $95 \%$ confidence interval (CI) of aTGCs parameters are derived using statistics tools, such as $\Delta \log$-likelihood, Bayesian or Frequentist methods. Results are presented in the following: Figure 3 shows the CI derived for aTGCs parameters in $W W Z$ and $W W \gamma$ vertices through $W W, W Z$ and $W \gamma$ production $[11,13,15]$. Figure 4 shows the limits for aTGCs parameters in $Z Z Z$ and $Z Z \gamma$ vertices through $Z Z$ production $[20,22]$. Figure 5 shows the limits for aTGCs parameters in $Z Z \gamma$ and $Z \gamma \gamma$ vertices through $Z \gamma$ production $[11,12]$. Results show consistency with SM couplings, therefore no anomaly is observed. The limits for aTGCs derived here are more stringent than those derived at the Tevatron, and in certain channels these are better than those from LEP.

\section{Summary}

Thanks to the very successful running of LHC and smooth operations of the ATLAS and CMS detectors, the diboson production cross-sections are measured in many channels and found to be consistent with the SM predictions. Data agree with SM couplings in aTGCs searches, and the derived $95 \%$ CI limits are improving existing results from Tevatron and are comparable with those from LEP.

\section{References}

[1] ATLAS Collaboration, JINST 3, S08003 (2008)

[2] CMS Collaboration, JINST 3, S08004 (2008)

[3] John M. Campbell etc., JHEP 07, 018 (2011)

[4] Mark S. Neubauer, Annu.Rev.Nucl.Part.Sci. 61, 223-250 (2011)

[5] Perelstein M.Prog., Part.Nucl.Phys. 58, 247 (2007)

[6] Lane K., Phys.Rev.D 60, 075007 (1999)

[7] Pati JC etc., Phys.Rev.D 10, 275 (1974)

[8] Randall L. and Sundrum R., Phys.Rev.Lett. 83, 3370 (1999)

[9] https://twiki.cern.ch/twiki/bin/view/AtlasPublic/ CombinedSummaryPlots

[10] https://twiki.cern.ch/twiki/bin/view/CMSPublic/ PhysicsResultsEWK

[11] ATLAS collaboration, Phys.Lett.B 717, 49 (2012)

[12] https://twiki.cern.ch/twiki/bin/view/CMSPublic/ PhysicsResultsEWK11009

[13] ATLAS collaboration, Phys.Lett.B 712, 289-308 (2012)

[14] CMS-PAS-SMP-12-005,CMS-PAS-SMP-12-013

[15] CERN-PH-EP-2012-179

[16] CMS-PAS-EWK-11-010

[17] ATLAS-CONF-2012-157

[18] CMS collaboration, CERN-PH-EP-2012-311

[19] ATLAS-CONF-2012-026,ATLAS-CONF-2012-090

[20] CMS collaboration, CERN-PH-EP-2012-336, CMS-PAS-SMP-12-014

[21] ATLAS-CONF-2012-027

[22] CERN-PH-EP-2012-318 
Table 1. The measured $W W$ cross-sections in ATLAS and CMS (referenced from Table 6 of [13] and Section 7 of [14]).

\begin{tabular}{lllll}
\hline Experiment & $\sqrt{s}$ & $\int L\left(\mathrm{fb}^{-1}\right)$ & $\sigma(p p \rightarrow W W)(\mathrm{pb})$ & SM NLO prediction $(\mathrm{pb})$ \\
\hline ATLAS & $7 \mathrm{TeV}$ & 4.6 & $51.9 \pm 2.0$ (stats. $) \pm 3.9$ (syst.) \pm 2.0 (lumi.) & $44.7_{-1.9}^{+2.1}$ \\
CMS & $7 \mathrm{TeV}$ & 4.9 & $52.5 \pm 2.0$ (stats.) \pm 4.5 (syst.) \pm 1.2 (lumi.) & - \\
CMS & $8 \mathrm{TeV}$ & 3.5 & $69.9 \pm 2.8$ (stats.) \pm 5.6 (syst.) \pm 3.1 (lumi.) & $57.3_{-1.6}^{+2.4}$ \\
\hline
\end{tabular}

Table 2. The measured $W Z$ cross-sections in ATLAS and CMS (referenced from Section 6.1 of [15] and Section 5.4 of [16]).

\begin{tabular}{lllll}
\hline Experiment & $\sqrt{s}$ & $\int L\left(\mathrm{fb}^{-1}\right)$ & $\sigma(p p \rightarrow W Z)(\mathrm{pb})$ & SM NLO prediction $(\mathrm{pb})$ \\
\hline ATLAS & $7 \mathrm{TeV}$ & 4.6 & $19.0_{-1.3}^{+1.4}$ (stats.) \pm 0.9 (syst.) $\pm 0.4($ lumi. $)$ & $17.6_{-1.0}^{+1.1}$ \\
CMS & $7 \mathrm{TeV}$ & 1.1 & $17.0 \pm 2.4$ (stats. \pm 1.1 (syst.) $\pm 1.0($ lumi. $)$ & - \\
\hline
\end{tabular}

Table 3. The measured $W W+W Z$ cross-sections in ATLAS and CMS through semi-leptonic decay channels (referenced from Section 7 of [17] and Page 5 of [18]).

\begin{tabular}{lllll}
\hline Experiment & $\sqrt{s}$ & $\int L\left(\mathrm{fb}^{-1}\right)$ & $\sigma(p p \rightarrow W W+W Z)(\mathrm{pb})$ & SM NLO prediction $(\mathrm{pb})$ \\
\hline ATLAS & $7 \mathrm{TeV}$ & 4.6 & $72 \pm 9$ (stats. $) \pm 15$ (syst.) \pm 13 (MC stats.) & $63.4 \pm 2.6$ \\
CMS & $7 \mathrm{TeV}$ & 5.0 & $68.9 \pm 8.7$ (stats. \pm 9.7(syst.) \pm 1.5 (lumi.) & - \\
\hline
\end{tabular}

Table 4. The measured ZZ cross-sections in ATLAS and CMS through fully leptonic decay channels (referenced from Section 6 of [19] and Section 7 of [20]).

\begin{tabular}{lllll}
\hline Experiment & $\sqrt{s}$ & $\int L\left(\mathrm{fb}^{-1}\right)$ & $\sigma(p p \rightarrow Z Z)(\mathrm{pb})$ & SM NLO prediction $(\mathrm{pb})$ \\
\hline ATLAS & $7 \mathrm{TeV}$ & 4.6 & $7.2_{-0.9}^{+1.1}$ (stat. $)_{-0.3}^{+0.4}$ (syst.) \pm 0.3 (lumi) & $6.5_{-0.2}^{+0.3}$ \\
CMS & $7 \mathrm{TeV}$ & 5.0 & $6.2_{-0.8}^{+0.9}$ (stat. $)_{-0.3}^{+0.4}$ (syst. \pm 0.1 (lumi) & - \\
ATLAS & $8 \mathrm{TeV}$ & 5.8 & $9.3_{-1.1}^{+1.0}(\text { (stat. })_{-0.3}^{+0.4}$ (syst. \pm 0.3 (lumi) & $7.4 \pm 0.4$ \\
CMS & $8 \mathrm{TeV}$ & 5.3 & $8.4 \pm 1.0$ (stats.) \pm 0.7 (syst. $) \pm 0.4$ (lumi.) & - \\
\hline
\end{tabular}



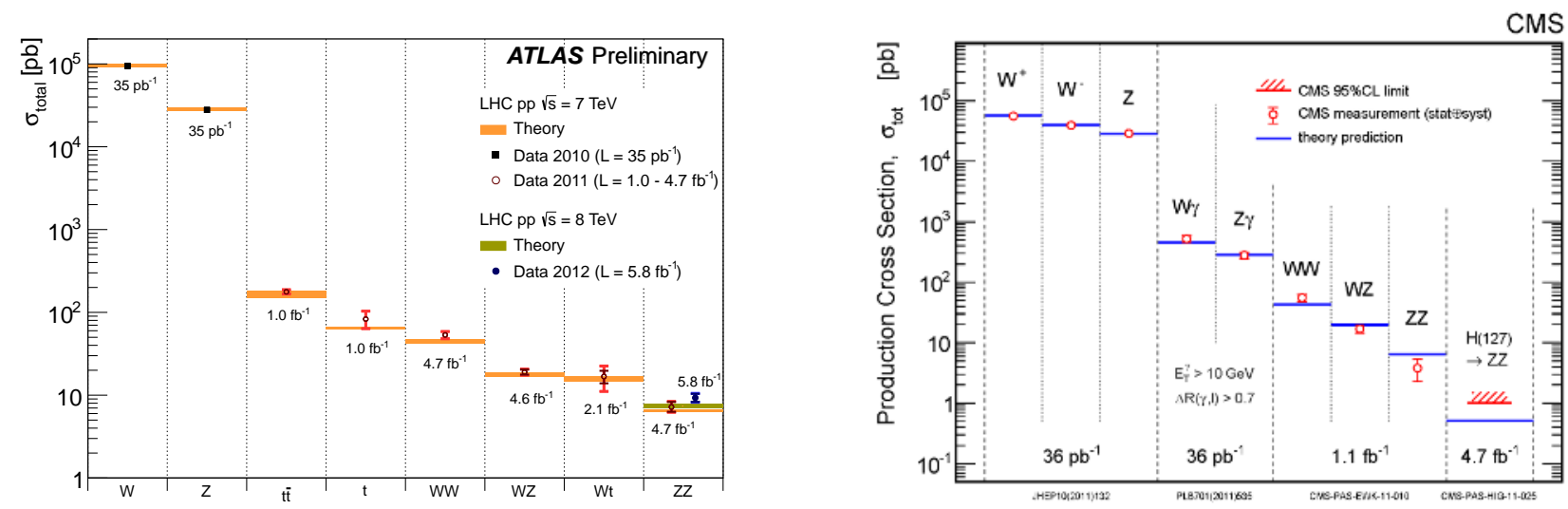

Figure 1. Summary plot for diboson cross-section measurement in ATLAS (left, from public twiki [9]) and CMS (right, from public twiki [10]).
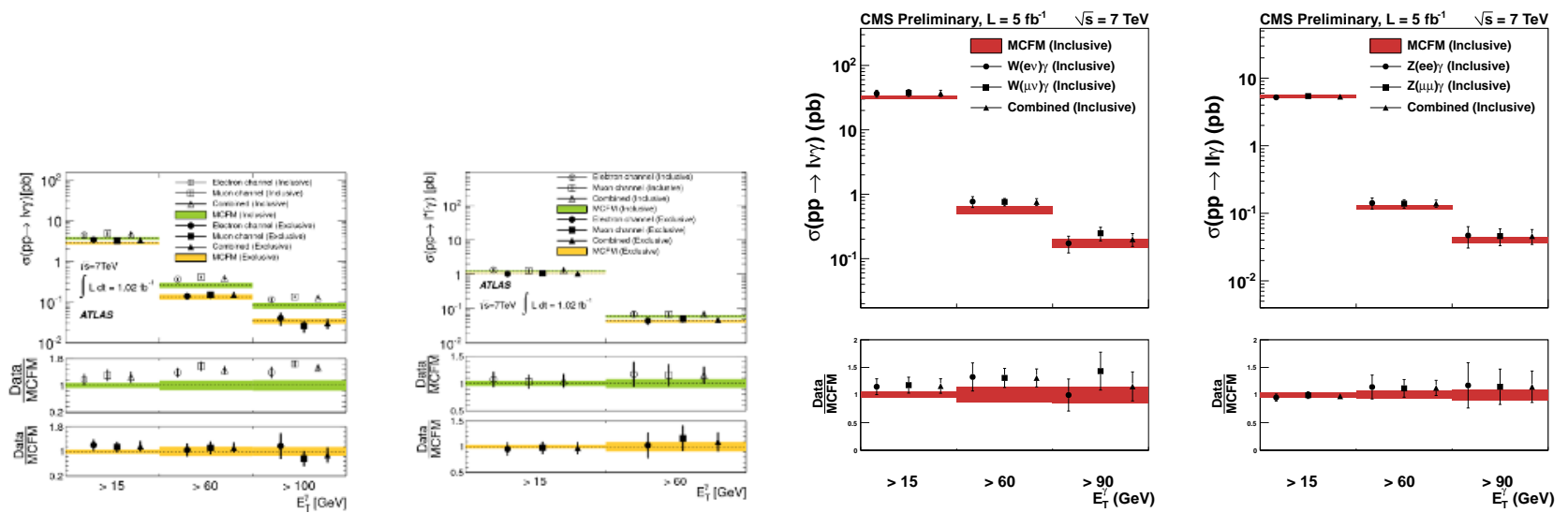

Figure 2. Measured production cross-sections and comparisons to SM NLO predictions for $W+\gamma$ in ATLAS (left), $Z+\gamma$ in ATLAS (middle left, from Figure 3 of [11]), $W+\gamma$ in CMS (middle right) and $Z+\gamma$ in CMS (right, from public twiki [12])
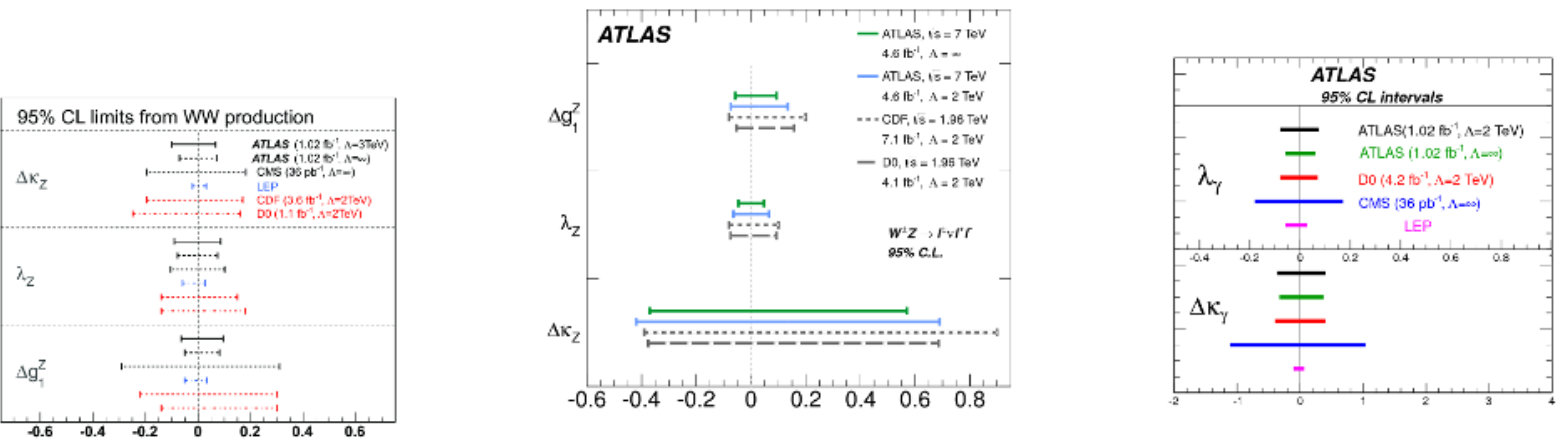

Figure 3. The 95\% CI of aTGCs parameters in $W W Z$ and $W W \gamma$ vertices derived from $W W$ (left, from Figure 10 of [13]), $W Z$ (middle, from Figure 5 of [15]) and $W \gamma$ (right, from Figure 4 of [11]) productions. 

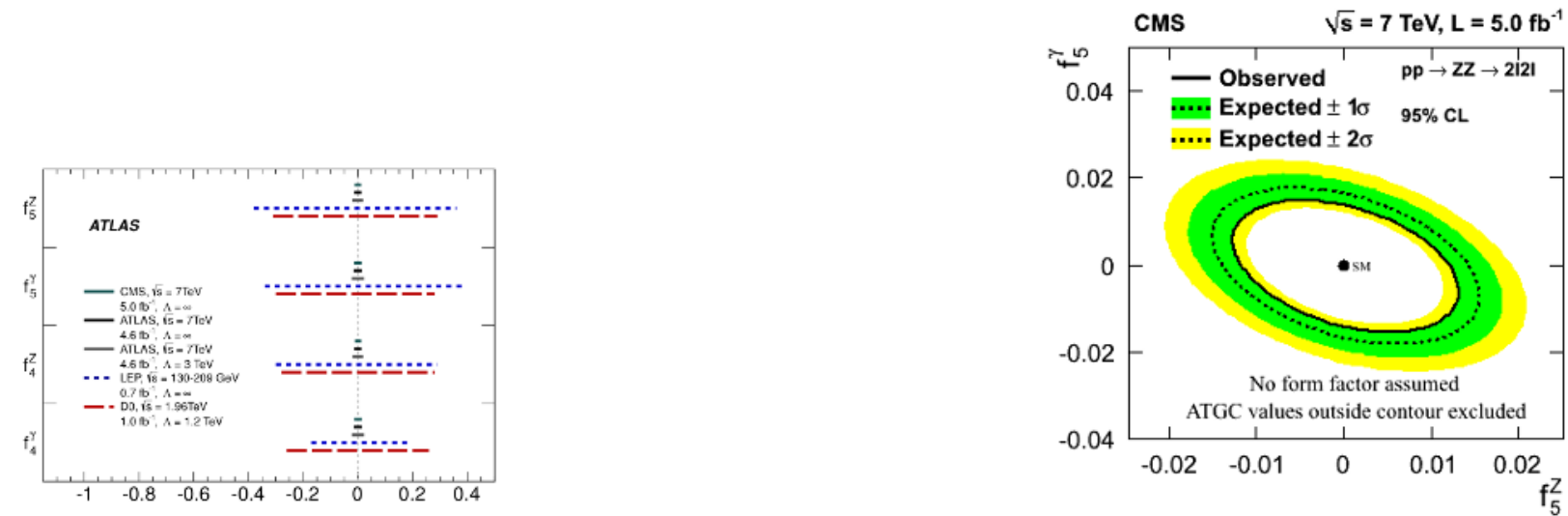

Figure 4. The $95 \%$ CI of aTGCs parameters in $Z Z Z$ and $Z Z \gamma$ vertices derived from $Z Z$ production in ATLAS (left, from Figure 13 of [19]), CMS (right, from Figure 3 of [20]).
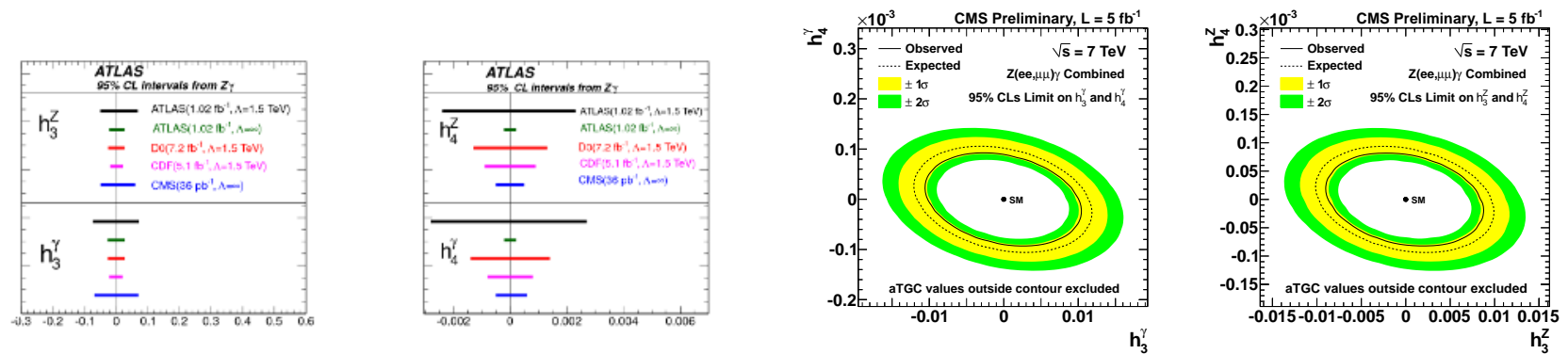

Figure 5. The 95\% CI of aTGCs parameters in $Z Z \gamma$ and $Z \gamma \gamma$ vertices derived from $Z \gamma$ production in ATLAS (left two, from Figure 4 of [11]) and CMS (right two, from public twiki [12]). 